\title{
Study of momentum space scalar amplitudes in AdS spacetime
}

\author{
Soner Albayrak $\odot,{ }^{1,2}$ Chandramouli Chowdhury, ${ }^{3}$ and Savan Kharel $\odot^{4}$ \\ ${ }^{1}$ Department of Physics, Yale University, New Haven, Connecticut 06511, USA \\ ${ }^{2}$ Walter Burke Institute for Theoretical Physics, Caltech, Pasadena, California 91125, USA \\ ${ }^{3}$ International Centre for Theoretical Sciences, Tata Institute of Fundamental Research, \\ Sivakote, Bangalore 560089, India \\ ${ }^{4}$ Department of Physics, Williams College, Williamstown, Massachusetts 01267, USA
}

(Received 1 April 2020; accepted 5 June 2020; published 22 June 2020)

\begin{abstract}
In this paper, we explore momentum space approach to computing scalar amplitudes in anti-de Sitter (AdS) space. We show that the algorithm derived by Arkani-Hamed, Benincasa, and Postnikov for cosmological wave functions can be straightforwardly adopted for AdS transition amplitudes in momentum space, allowing one to bypass bulk point integrations. We demonstrate the utility of this approach in AdS by presenting several explicit results both at tree and loop level.
\end{abstract}

DOI: 10.1103/PhysRevD.101.124043

\section{INTRODUCTION}

In the last decade, there has been a resurrection in the study of scattering amplitudes and conformal correlation functions. Such undertakings have extricated rich structures of quantum field theory and quantum gravity. In particular, we now have considerable evidence that scattering amplitudes in quantum gravity can be computed from the correlation functions in one lower dimensions. Such a correspondence is known as the holographic duality [1-3] and its most concrete formalism in given by AdS/CFT where the bulk geometry is anti-de Sitter space and the conformal correlation functions live at the boundary. This correspondence has led to major insights into the nature of quantum gravity as well as gauge theory.

Besides yielding useful insights, holographic correlators have been discovered to have rich mathematical structures [4-32]. However, momentum-space methods, our usual modus operandi of doing computation in quantum field theory, is still not yet fully studied for CFTs (see for a partial list of progress [33-58]). We also now know that the usual scattering amplitudes arise from the flat space limit of the holographic Conformal Field Theory (CFT) correlators. Hence it is useful to generalize the tools that have been developed for the usual scattering amplitudes in flat space to holographic correlators. A modest step in this direction is taken in [59] where it is shown that tree level gauge theory Witten diagrams for transition amplitudes, reduce to

Published by the American Physical Society under the terms of the Creative Commons Attribution 4.0 International license. Further distribution of this work must maintain attribution to the author(s) and the published article's title, journal citation, and DOI. Funded by SCOAP ${ }^{3}$. surprisingly simple expressions when expressed in momentum space. ${ }^{1}$ In a similar vein, momentum space approach to transition amplitudes also simplifies the computation of graviton exchange diagrams, which was demonstrated in [60] with explicit higher point tree level results.

The momentum space formalism for AdS calculations can be upgraded with a new algorithm developed by Arkani-Hamed, Benincasa, and Postnikov in [61] where they investigate the wave function of the Universe in de Sitter background. Indeed, it was realized in [62] that the computation of gluon Witten diagrams in $\mathrm{AdS}_{4}$ can actually use the same combinatorial relations developed in [61] if it is written in momentum space. This correspondence allowed the authors to compute any tree level gluon exchange diagram algebraically, without having to do any explicit bulk integrations.

In this paper, we would like to extend this marriage between Arkani-Hamed et al.'s algorithm and AdS momentum space beyond $\mathrm{AdS}_{4}$ gluons. We will show that conformally coupled scalars in any $\mathrm{AdS}_{d+1}$ can be computed algorithmically, both at tree and loop level, and we will demonstrate this with explicit results for various Witten diagrams. Besides its formal usage, the AdS transition amplitudes can be useful in the computation of the wave function at late times from which one can compute de Sitter correlators $[63,64]$. The growing interest in cosmology has generated a great deal of excitement in the study of late time

\footnotetext{
${ }^{1}$ Transition amplitudes are generalizations of vacuum-correlators such that one replaces some of the bulk to boundary propagators of the relevant Witten diagram with normalizable modes [37]. Such a replacement roughly creates the effect of changing the boundary conditions at past and future horizons of the relevant Poincaré patch, creating past and future states for the correlator (hence the name transition amplitude).
} 
de Sitter correlators [38,50,65-84] and we believe that the analogous calculations of momentum space AdS amplitudes can assist in the study of the shape of nonGaussianities.

Here is the organization of the paper. In Sec. II, we discuss scalars in curved spacetime and present the review of momentum space toolkit in anti-de Sitter space. We also demonstrate the standard nonalgorithmic approach of momentum space formalism by computing Witten diagrams for minimally coupled scalars. In Sec. III, we switch to conformally coupled scalars, discuss how the algorithmic approach works, and provide explicit results both at tree and loop levels. Finally, we conclude with a brief discussion and future directions.

\section{PRELIMINARIES}

\section{A. Scalars in curved spacetime}

A free scalar $\phi$ in flat space satisfies the Klein-Gordon equation which reads as

$$
\left(\square-m^{2}\right) \Phi=0
$$

where $m$ is the mass parameter of the field. In curved spacetime, this equation becomes

$$
\left(\square-\left(m^{2}+\xi R\right)\right) \Phi=0
$$

where $R$ is the Ricci scalar and $\xi$ is a coefficient determining the interaction between scalar and the background. In the case of $\mathrm{AdS}_{d+1}$, this equation follows from the action

$$
\begin{aligned}
S_{\text {quadratic }}= & -\frac{1}{2} \int d^{d+1} x \sqrt{g}\left(g^{\mu \nu}\left(\partial_{\mu} \Phi\right)\left(\partial_{\nu} \Phi\right)\right. \\
& \left.+\left(m^{2}+\xi R\right) \Phi^{2}\right)
\end{aligned}
$$

where $g=\left|\operatorname{det} g_{\mu \nu}\right|$ and we stick to mostly positive metric convention throughout the paper.

The scalar in curved spacetime has been extensively analyzed in the literature; however, the analysis usually focus on two specific values of $\xi$ : minimally coupled scalar with $\xi \rightarrow 0$, and conformally coupled scalar with $\xi \rightarrow \xi_{c}$ for

$$
\xi_{c} \equiv \frac{d-1}{4 d} .
$$

The popularity of minimally coupled scalar follows from the fact that $\xi=0$ simplifies the Lagrangian. The appeal of conformally coupled scalar, however, cannot be immediately seen unless one goes to local Minkowski frame where the potential term takes the form [85]

$$
V(x) \sim\left[m^{2}+\left(\xi-\xi_{c}\right) R\right]
$$

One sees that if (in addition to $\xi=\xi_{c}$ ) one imposes $m=0$, the potential vanishes, leading the theory to enjoy conformal symmetry. Indeed, even though all pairs $(m, \xi)=$ $\left(m, \xi_{c}\right)$ fall into the class of conformally coupled scalars, only $(m, \xi)=\left(0, \xi_{c}\right)$ case is invariant under conformal transformations [86].

Tuning the parameters $(m, \xi)=\left(0, \xi_{c}\right)$ is necessary for the theory to enjoy conformal symmetry but it is not sufficient: we also need to check if the interaction Lagrangian spoils this symmetry. As a prerequisite condition of scale invariance we restrict to interactions of the form $\mathcal{O} \equiv \mathcal{O}\left(g^{\mu \nu}, \partial_{\mu}, \Phi\right)$ which transforms as $\mathcal{O} \rightarrow \lambda^{\kappa} \mathcal{O}$ as $g_{\mu \nu} \rightarrow \lambda^{2} g_{\mu \nu}$ for constant $\lambda$.

In this paper, we focus on nonderivative interactions for which the action takes the form

$$
\begin{aligned}
S= & -\int d^{d+1} x \sqrt{g}\left[\frac { 1 } { 2 } \left(g^{\mu \nu}\left(\partial_{\mu} \Phi\right)\left(\partial_{\nu} \Phi\right)\right.\right. \\
& \left.\left.+\left(m^{2}+\xi R\right) \Phi^{2}\right)+\frac{\lambda_{n}}{n !} \Phi^{n}\right] .
\end{aligned}
$$

We can check the trace of stress tensor $T^{\mu \nu}$ to see when it is zero. Indeed, via

$$
T_{\mu \nu}=\frac{-2}{\sqrt{g}} \frac{\delta S}{\delta g^{\mu \nu}}
$$

we obtain

$$
\begin{aligned}
T_{\mu \nu}= & \left(\partial_{\alpha} \Phi\right)\left(\partial_{\beta} \Phi\right)\left[\delta_{\mu}^{\alpha} \delta_{\nu}^{\beta}-\frac{1}{2} g_{\mu \nu} g^{\alpha \beta}\right]-\xi\left[\partial_{\mu} \partial_{\nu}-g_{\mu \nu} \square\right] \Phi^{2} \\
& -\frac{1}{2} \Phi^{2}\left[\left(m^{2}+\xi R\right) g_{\mu \nu}-2 \xi R_{\mu \nu}\right]-\frac{\lambda_{n}}{n !} \Phi^{n} g_{\mu \nu}
\end{aligned}
$$

where we used

$\delta F(R)=\left(F^{\prime}(R) R_{\mu \nu}-\left[\nabla_{\mu} \nabla_{\nu}-g_{\mu \nu} \square\right] F^{\prime}(R)\right) \delta g^{\mu \nu}$,

for whose derivation with a nice explanation we suggest the lecture notes of Matthias Blau, available at http://www.blau .itp.unibe.ch/newlecturesGR.pdf. ${ }^{2}$

\footnotetext{
${ }^{2}$ This equation is actually true only if there are no contributions at the boundary as its derivation uses integration by parts and assumes that total derivative terms do not contribute. Strictly speaking, this is not correct for AdS. However, boundary conditions actually kill the additional piece unless the variation of the action on the boundary contains the derivative of the variation of the boundary metric for which one then needs to add an appropriate boundary term to cancel the additional variation, see [87] in the case of Einstein gravity. We will avoid such subtleties (and refer the reader to $[88,89]$ and references therein) so a rigorous minded reader should see our calculations not as a derivation but as a motivation for why only certain interactions can enjoy full conformal symmetry.
} 
The trace of stress tensor then reads as

$$
\begin{aligned}
g^{\mu \nu} T_{\mu \nu}= & 2 d\left(\xi-\xi_{c}\right) g^{\mu \nu}\left(\partial_{\mu} \Phi\right)\left(\partial_{\nu} \Phi\right) \\
& +2 d \xi \Phi\left[\square \Phi-\left(\frac{d+1}{d-1} m^{2}+\xi_{c} R\right) \Phi\right. \\
& \left.-\frac{\lambda_{n}}{n !} \frac{\xi_{c}}{\xi} \frac{2(d+1)}{d-1} \Phi^{n-1}\right]
\end{aligned}
$$

We see the first term dies only if $\xi=\xi_{c}$. We can then kill the second term with equation of motion if $m=0$ and $n=n_{c}$ for

$$
n_{c} \equiv \frac{2(d+1)}{d-1}
$$

e.g., $n_{c}=4$ for $\mathrm{AdS}_{4}$. We thus arrived at the well-known conclusion: the action in Eq. (2.6) enjoys conformal symmetry only if $\{m, \xi, n\}=\left\{0, \xi_{c}, n_{c}\right\}$.

We can derive this result from another, and slightly simpler, approach. We first specialize to the AdS with the Poincaré metric and the Ricci scalar

$$
d s^{2}=\frac{d z^{2}+\eta_{i j} d x^{i} d x^{j}}{(z / \rho)^{2}}, \quad R=-\frac{d(d+1)}{\rho^{2}}
$$

where we take AdS radius $\rho=1$ in the rest of the paper. ${ }^{3}$ We then consider a Weyl transformation which maps AdS to flat space:

$$
\begin{gathered}
g_{\mu \nu} \rightarrow g_{\mu \nu}^{\prime} \equiv z^{2} g_{\mu \nu} \\
\Phi \rightarrow \phi \equiv z^{-\frac{d-1}{2}} \Phi
\end{gathered}
$$

where we used the engineering scaling dimension for the scalar field. Under this transformation, quadratic part which is invariant under conformal transformations map to the free scalar in flat space

$\frac{1}{2} \sqrt{g} g^{\mu \nu}\left(\partial_{\mu} \Phi\right)\left(\partial_{\nu} \Phi\right)+\frac{1}{2} \xi \sqrt{g} R \Phi^{2} \rightarrow \frac{1}{2}\left(\partial_{\mu} \phi\right)\left(\partial^{\mu} \phi\right)$

whereas the interaction part maps as

$$
\sqrt{g} \frac{\lambda_{n}}{n !} \Phi^{n} \rightarrow z^{\frac{d-1}{2}\left(n-n_{c}\right)} \frac{\lambda_{n}}{n !} \phi^{n}
$$

\footnotetext{
${ }^{3}$ Our notation is such that $z$ is the radial coordinate and the transverse coordinates $x_{i}$ approach to the boundary coordinate as $z \rightarrow 0$.
}

We immediately see that we need $n=n_{c}$ if we require the flat space interaction to be conformally invariant as well.

\section{B. Review of momentum space toolkit in AdS}

In this section, we will review the basics of our framework and specifics regarding the scalars. For similar reviews in the context of gauge fields and gravitons, see $[59,60,62]$.

We will be working with the Poincaré patch of Eq. (2.12) and take the Fourier transform of $x_{i}$. We will leave $z$ as it is though: the coordinates $\left\{z, k_{i}\right\}$ are what we call the momentum space in this paper. This is in the same spirit of the treatment in $[33,35,37]$.

In momentum space, equation of motion in Eq. (2.2) becomes

$$
\left(z^{1+d} \partial_{z} z^{1-d} \partial_{z}-z^{2} k_{i} k^{i}-\mu^{2}\right) \Phi\left(z, k_{i}\right)=0
$$

where we define the effective mass square

$$
\mu^{2} \equiv m^{2}+\xi R
$$

The general solution to this differential equation for timelike momenta $k_{i} k^{i}<0$ reads as: ${ }^{4}$

$$
\Phi(z) \sim c_{1} z^{d / 2} J_{\nu}(k z)+c_{2} z^{d / 2} Y_{\nu}(k z)
$$

where we define

$$
\nu \equiv \frac{\sqrt{d^{2}+4 \mu^{2}}}{2}
$$

and where $k \equiv \sqrt{\left|k_{i} k^{i}\right|}$. $^{5,6}$

For spacelike momenta, the regularity in the AdS can only be achieved for the particular combination which sums up to the Bessel function of the second kind, i.e.,

$$
\Phi_{k}(z) \sim z^{d / 2} K_{\nu}(k z)
$$

\footnotetext{
${ }^{4}$ Here, $J_{n}(x)\left(Y_{n}(x)\right)$ is the Bessel function of the first (second) kind.

${ }^{5}$ We specifically chose the letter $\nu$ to denote $\frac{\sqrt{d^{2}+4 \mu^{2}}}{2}$ as this term up to an overall $i$ is the pole of the spectral representation of the bulk to bulk propagator, usually denoted as $\pm i \nu$ in the literature [90], where there are two poles due to the shadow symmetry.

${ }^{6}$ The scaling dimension of the dual operator in the boundary CFT is $\Delta=\frac{d}{2}+\nu$ in our notation. We would like to caution the reader that many papers in literature calls mass $m$ what we defined as the effective mass $\mu$, hence the well-known formula $\Delta(\Delta-d)=m^{2}$, which becomes $\Delta(\Delta-d)=\mu^{2}$ in our notation. As we choose to distinguish mass $m$ and effective mass $\mu$, it is completely consistent in our definition when we say massless conformally coupled scalar as $m=0$ despite $\mu^{2}=\frac{1-d^{2}}{4} \neq 0$.
} 
Note that this is always possible due to the identity ${ }^{7}$

$$
K_{\nu}(z)=\frac{\pi i^{\nu}}{2}\left(i J_{\nu}(i z)-Y_{\nu}(i z)\right)
$$

Below, we will focus on massless minimally coupled scalars $\Phi_{k}^{(m)}$ and massless conformally coupled scalars $\Phi_{k}^{(c)}$ for which the relevant bulk to boundary propagators read as

$$
\begin{aligned}
& \Phi_{k}^{(m)}(z) \sim z^{d / 2} K_{d / 2}(k z) \\
& \Phi_{k}^{(c)}(z) \sim z^{d / 2} K_{1 / 2}(k z)
\end{aligned}
$$

where we use the fact that Ricci scalar $R=-d(d+1)$ in AdS.

We can similarly calculate the bulk to bulk propagators. We are looking for the solutions to the equation

$$
\left(z^{1+d} \partial_{z} z^{1-d} \partial_{z}-z^{2} k_{i} k^{i}-\mu^{2}\right) G_{\Phi}\left(z, z^{\prime}, k_{i}\right)=i \delta\left(z-z^{\prime}\right) z^{d+1} .
$$

We observe that

$$
\begin{aligned}
& \left(\partial_{z} z^{1-d} \partial_{z}-z^{1-d} k_{i} k^{i}-z^{-1-d} \mu^{2}\right) \\
& \quad \times\left(-\frac{i p z^{d / 2} z^{\prime d / 2} J_{\nu}(p z) J_{\nu}\left(p z^{\prime}\right)}{k_{i} k^{i}+p^{2}}\right) \\
& \quad=i z^{1-d / 2} z^{\prime d / 2} p J_{\nu}(p z) J_{\nu}\left(p z^{\prime}\right)
\end{aligned}
$$

and since we also have the identity

$$
\int_{0}^{\infty} p J_{\nu}(p z) J_{\nu}\left(p z^{\prime}\right) d p=\frac{\delta\left(z-z^{\prime}\right)}{z}
$$

we find the propagator:

$$
\begin{aligned}
G_{\Phi}\left(z, z^{\prime}, k_{i}\right)= & \int_{0}^{\infty} \frac{-i p d p}{k_{i} k^{i}+p^{2}-i \epsilon} \\
& \left(z^{d / 2} J_{\nu}(p z)\right)\left(z^{\prime d / 2} J_{\nu}\left(p z^{\prime}\right)\right) .
\end{aligned}
$$

In particular, $\nu \rightarrow 1 / 2(d / 2)$ gives the propagator for conformally (minimally) coupled scalar as we noted above.

One can now go ahead and write the expression for Witten diagrams. At tree level, the amplitude for a diagram of $m$ external legs and $n$ bulk propagators reads as

$$
\begin{aligned}
W_{m, n} \sim & \int_{0}^{\infty} d z_{1} \ldots d z_{n+1} \Phi_{k_{1}}\left(z_{i_{1}}\right) \ldots \Phi_{k_{m}}\left(z_{i_{m}}\right) \\
& \times G_{\Phi}\left(z_{j_{1}}, z_{j_{2}}, q_{1}\right) \ldots G_{\Phi}\left(z_{j_{n}}, z_{j_{n}}, q_{n}\right) \prod_{t=1}^{n+1} \rho_{t}\left(z_{t}\right)
\end{aligned}
$$

for the interaction coefficient $\rho_{n}\left(z_{n}\right)$ at $n$th vertex, where $z_{i_{1}}, \ldots, z_{i_{m}} \in\left\{z_{1}, \ldots, z_{n+1}\right\} \quad$ and $\quad z_{j_{1}}, \ldots, z_{j_{r}} \in\left\{z_{1}, \ldots\right.$, $\left.z_{n+1}\right\}$, and where $q_{i}$ are norms of linear combinations of vectors $\boldsymbol{k}_{i}$ depending on the topology.

As an example, we can consider the topology in Fig. 1 for minimally coupled scalars; the relevant amplitude would read as

$$
\begin{aligned}
W \sim & \int_{0}^{\infty} d z_{1} d z_{2} \Phi_{k_{1}}^{(m)}\left(z_{1}\right) \Phi_{k_{2}}^{(m)}\left(z_{1}\right) \Phi_{k_{3}}^{(m)}\left(z_{2}\right) \Phi_{k_{4}}^{(m)} \\
& \times\left(z_{2}\right) \Phi_{k_{5}}^{(m)}\left(z_{2}\right) G_{\phi}\left(z_{1}, z_{2}, k_{\underline{12}}\right) \lambda_{3} \lambda_{4}
\end{aligned}
$$

where we are following the notation of [59] for addition of $k$-vectors. ${ }^{8}$

We can use Eq. (2.26) and exchange the order of integrations, writing Eq. (2.27) in a different form:

${ }^{7}$ This is only true for $z>0$. For generic $z$, the relevant identity reads as

$$
K_{\nu}(z)= \begin{cases}i^{\nu}\left((-\log (z)+\log (i z)) J_{\nu}(i z)-\frac{1}{2} \pi Y_{\nu}(i z)\right) & \text { for } \nu \in \mathbb{Z} \\ \frac{1}{2} \pi \csc (\pi \nu)\left(\cos (\pi \nu)(i z)^{\nu} z^{-\nu}-(i z)^{-\nu} z^{\nu}\right) J_{\nu}(i z)-\frac{1}{2} \pi(i z)^{\nu} z^{-\nu} Y_{\nu}(i z) & \text { for } \nu \notin \mathbb{Z}\end{cases}
$$

${ }^{8}$ Explicitly:

$$
k_{i_{11} i_{12} \ldots i_{1 n_{1}}} \underline{i_{21} i_{22} \ldots i_{2 n_{2}} \ldots i_{m 1} i_{m 2} \ldots i_{m m_{m}}} j_{1} j_{2} \ldots j_{p}:=\sum_{a=1}^{m}\left|\sum_{b=1}^{n_{a}} \boldsymbol{k}_{i_{a b}}\right|+\sum_{c=1}^{p}\left|\boldsymbol{k}_{j_{c}}\right|,
$$

and

$$
\boldsymbol{k}_{i_{1} i_{2} \ldots i_{n}}:=\boldsymbol{k}_{i_{1}}+\boldsymbol{k}_{i_{2}}+\cdots+\boldsymbol{k}_{i_{n}} .
$$

For example, $k_{12345} \equiv\left|\boldsymbol{k}_{1}+\boldsymbol{k}_{2}\right|+\left|\boldsymbol{k}_{3}\right|+\left|\boldsymbol{k}_{4}+\boldsymbol{k}_{5}\right|$ and $\boldsymbol{k}_{12} \equiv \boldsymbol{k}_{1}+\boldsymbol{k}_{2}$. 


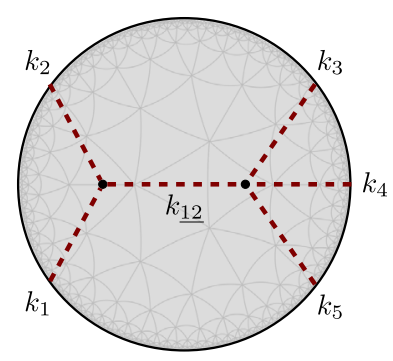

FIG. 1. A five point tree level Witten diagram, labeled as $W_{5,1}$ below.

$$
\begin{aligned}
W_{m, n} \sim & \int_{0}^{\infty} d p_{1} \ldots d p_{n} \frac{-i p_{1}}{p_{1}^{2}+q_{1}^{2}-i \epsilon} \ldots \frac{-i p_{n}}{p_{n}^{2}+q_{n}^{2}-i \epsilon} \\
& \times \mathcal{B}_{1}\left(k_{i}, p_{i}\right) \ldots \mathcal{B}_{n+1}\left(k_{i}, p_{i}\right) \prod_{t=1}^{n+1} \rho_{t}
\end{aligned}
$$

where $\mathcal{B}$ is what we will call bulk-point integrated expression. Note that we integrated (if any) $z$-dependent parts of the interaction coefficients as well.

\section{Example: Minimally coupled scalars in $\mathbf{A d S}_{\mathbf{4}}$}

In this section we will calculate some amplitudes for tree level Witten diagrams using the procedure advocated in the previous section; specifically, with

$$
\begin{aligned}
\phi_{k}(z) \equiv & \sqrt{\frac{2}{\pi}}(k z)^{3 / 2} K_{3 / 2}(k z) \\
G_{\phi}\left(z, z^{\prime}, k_{i}\right) \equiv & \int_{0}^{\infty} \frac{-i p d p}{k_{i} k^{i}+p^{2}-i \epsilon} \\
& \times\left(z^{3 / 2} J_{3 / 2}(p z)\right)\left(z^{\prime 3 / 2} J_{3 / 2}\left(p z^{\prime}\right)\right)
\end{aligned}
$$

where we choose a particular normalization for the bulk to boundary propagator consistent with the literature. ${ }^{9}$

Apart from its physical significance, we focus on $\mathrm{AdS}_{4}$ also because Bessel functions simplify for half integer arguments; hence the calculations are relatively easier for $\mathrm{AdS}_{2+2 n}$. This motivation was also used in previous similar work $[59,60,62]$, where the calculation of graviton amplitudes in [60] is actually quite similar to the computation at hand. Specially, one can write the graviton propagators in $\operatorname{AdS}_{d+1}$ as

$$
\begin{aligned}
h_{i j}\left(z, k_{i}\right) & =\frac{\epsilon_{i j}}{z^{2}} \phi_{k}(z) \\
G_{a b, c d}^{\text {graviton }}\left(z, z^{\prime}, k_{i}\right) & =\frac{i}{\left(z z^{\prime}\right)^{2}} \mathcal{D}_{a b, c d}^{\mathbf{k}} G_{\phi}\left(z, z^{\prime}, k_{i}\right)
\end{aligned}
$$

\footnotetext{
${ }^{9}$ An overall $k$-dependent scaling of bulk to boundary propagators, i.e. $\phi_{k}(z) \rightarrow f(k) \phi_{k}(z)$, is immaterial for our purposes in this paper, hence we refer this as a normalization and fix it with a convenient factor. However, this normalization is actually tightly constrained by scaling dimensions of the dual operators at the boundary CFT.
}

where $\mathcal{D}_{a b, c d}^{\mathbf{k}}$ is a differential operator whose details are irrelevant for us. However, one important remark is that these differential operators commute with the rest of the calculation, thus for a Witten diagram with $n$ bulk to bulk propagators we schematically have

$$
\mathcal{A}_{\text {graviton }}=\left(\epsilon_{i}, V_{i}\right)^{a_{11} a_{12} \ldots a_{14} a_{21} \ldots a_{n 4}} \prod_{j=1}^{n} \mathcal{D}_{a_{j 1} a_{j 2}, a_{j 3} a_{j 4}}^{p_{j}} \mathcal{M},
$$

where $\left(\epsilon_{i}, V_{i}\right)$ stand for the collection of the vertex factors and polarization vectors, $\boldsymbol{p}_{j}$ is the sum of some bulk to boundary momenta depending on the topology of the diagram, and $\mathcal{M}$ is the scalar factor of the amplitude. This scalar factor for graviton amplitude is almost the same expression with the amplitude for the same Witten diagram with graviton legs replaced by minimally coupled scalars. The only difference between the amplitude for minimally coupled scalars and graviton scalar factor is due to the different overall exponent of $z$ in bulk point integration. ${ }^{10,11}$

We now proceed with calculation of bulk point integrated expressions. Specifically, we define

$$
\begin{aligned}
\mathcal{K} \mathcal{K} \mathcal{K}\left(k_{1}, k_{2}, k_{3}\right) \equiv & \int_{0}^{\infty} \frac{d z}{z^{4}} \phi_{k_{1}}(z) \phi_{k_{2}}(z) \phi_{k_{3}}(z) \\
\mathcal{K} \mathcal{K} \mathcal{J}\left(k_{1}, k_{2}, p\right) \equiv & \int_{0}^{\infty} \frac{d z}{z^{4}} \phi_{k_{1}}(z) \phi_{k_{2}}(z)\left(z^{3 / 2} J_{3 / 2}(p z)\right) \\
\mathcal{K} \mathcal{J} \mathcal{J}\left(k, p_{1}, p_{2}\right) \equiv & \int_{0}^{\infty} \frac{d z}{z^{4}} \phi_{k}(z)\left(z^{3 / 2} J_{3 / 2}\left(p_{1} z\right)\right) \\
& \times\left(z^{3 / 2} J_{3 / 2}\left(p_{2} z\right)\right) \\
\mathcal{K} \mathcal{K} \mathcal{K} \mathcal{J}\left(k_{1}, k_{2}, k_{3}, p\right) \equiv & \int_{0}^{\infty} \frac{d z}{z^{4}} \phi_{k_{1}}(z) \phi_{k_{2}}(z) \phi_{k_{3}} \\
& \times(z)\left(z^{3 / 2} J_{3 / 2}(p z)\right)
\end{aligned}
$$

We can define similar expressions for more complicated interactions, but we will restrict to the first few simplest tree level Witten diagrams. By regularizing the integrations we find

\footnotetext{
${ }^{10}$ Of course, the vertex coefficients are also different but that is an overall factor which can be easily accounted for.

${ }^{11}$ There are two cases where $z$-factors coincidentally match: minimally coupled scalars with two-derivative-cubic interaction (polynomial quartic interaction) have exactly the same $z$-factor with cubic (quartic) graviton interaction. We will not be making use of that correspondence though, as we are only interested in polynomial scalar interactions and as we do not know of any explicit result in the literature for momentum space Witten diagrams of pure quartic graviton interactions.
} 


$$
\begin{gathered}
\mathcal{K} \mathcal{K} \mathcal{K}\left(k_{1}, k_{2}, k_{3}\right)=\frac{1}{9}\left(k_{1}+k_{2}+k_{3}\right)^{3}-k_{1} k_{2} k_{3}+\frac{1}{3}\left(k_{1}^{3}+k_{2}^{3}+k_{3}^{3}\right)\left(-\log \left(k_{1}+k_{2}+k_{3}\right)-\gamma+1\right) \\
\mathcal{K} \mathcal{K} \mathcal{J}\left(k_{1}, k_{2}, p\right)=\frac{\sqrt{\frac{2}{\pi}}\left(k_{1}^{3}+k_{2}^{3}\right)\left(\tan ^{-1}\left(\frac{p}{k_{1}+k_{2}}\right)-\frac{p}{k_{1}+k_{2}}\right)}{3 p^{3 / 2}}-\frac{p^{3 / 2}\left(3 \log \left(\left(k_{1}+k_{2}\right)^{2}+p^{2}\right)+6 \gamma-8\right)}{9 \sqrt{2 \pi}} \\
\mathcal{K} \mathcal{J} \mathcal{J}\left(k, p_{1}, p_{2}\right)=\frac{k^{3} \tanh ^{-1}\left(\frac{2 p_{1} p_{2}}{k^{2}+p_{1}^{2}+p_{2}^{2}}\right)-2 k p_{1} p_{2}+\left(p_{2}^{3}-p_{1}^{3}\right) \tan ^{-1}\left(\frac{p_{1}-p_{2}}{k}\right)+\left(p_{1}^{3}+p_{2}^{3}\right) \tan ^{-1}\left(\frac{p_{1}+p_{2}}{k}\right)}{3 \pi\left(p_{1} p_{2}\right)^{3 / 2}} \\
\mathcal{K} \mathcal{K} \mathcal{K} \mathcal{J}\left(k_{1}, k_{2}, k_{3}, p\right)=\frac{\sqrt{\frac{2}{\pi}}\left(k_{1}^{3}+k_{2}^{3}+k_{3}^{3}\right) \tan ^{-1}\left(\frac{p}{k_{1}+k_{2}+k_{3}}\right)}{3 p^{3 / 2}}-\frac{p^{3 / 2}\left(3 \log \left(\left(k_{1}+k_{2}+k_{3}\right)^{2}+p^{2}\right)+6 \gamma-8\right)}{9 \sqrt{2 \pi}} \\
-\sqrt{\frac{2}{\pi p}\left(\frac{k_{1} k_{2} k_{3}\left(k_{1}+k_{2}+k_{3}\right)}{\left(k_{1}+k_{2}+k_{3}\right)^{2}+p^{2}}+\frac{k_{1}^{3}+k_{2}^{3}+k_{3}^{3}-3 k_{1} k_{2} k_{3}}{3\left(k_{1}+k_{2}+k_{3}\right)}\right)}
\end{gathered}
$$

where $\gamma$ is the Euler-gamma number.

We can now use the prescription of Eq. (2.30) to write down the amplitudes for various Witten diagrams:

$$
\begin{gathered}
W_{3,0}\left(k_{i}\right)=-i \lambda_{3} \mathcal{K K \mathcal { K }}\left(k_{1}, k_{2}, k_{3}\right) \\
W_{4,1}\left(k_{i}\right)=-\lambda_{3}^{2} \int_{0}^{\infty} d p \frac{-i p}{p^{2}+k_{12}^{2}-i \epsilon} \mathcal{K} \mathcal{K} \mathcal{J}\left(k_{1}, k_{2}, p\right) \mathcal{K} \mathcal{K} \mathcal{J}\left(k_{3}, k_{4}, p\right) \\
W_{5,1}\left(k_{i}\right)=-\lambda_{3} \lambda_{4} \int_{0}^{\infty} d p \frac{-i p}{p^{2}+k_{12}^{2}-i \epsilon} \mathcal{K} \mathcal{K} \mathcal{J}\left(k_{1}, k_{2}, p\right) \mathcal{K} \mathcal{K} \mathcal{K} \mathcal{J}\left(k_{3}, k_{4}, k_{5}, p\right) \\
W_{5,2}\left(k_{i}\right)=i \lambda_{3}^{3} \int_{0}^{\infty} d p_{1} d p_{2} \frac{-i p_{1}}{p_{1}^{2}+k_{12}^{2}-i \epsilon} \frac{-i p_{2}}{p_{2}^{2}+k_{\underline{45}}^{2}-i \epsilon} \times \mathcal{K} \mathcal{K} \mathcal{J}\left(k_{1}, k_{2}, p_{1}\right) \mathcal{K} \mathcal{J} \mathcal{J}\left(k_{3}, p_{1}, p_{2}\right) \mathcal{K} \mathcal{K} \mathcal{J}\left(k_{4}, k_{5}, p_{2}\right) \\
W_{6,1}\left(k_{i}\right)=-\lambda_{4}^{2} \int_{0}^{\infty} d p \frac{-i p}{p^{2}+k_{123}^{2}-i \epsilon} \mathcal{K} \mathcal{K} \mathcal{J}\left(k_{1}, k_{2}, k_{3}, p\right) \mathcal{K} \mathcal{K} \mathcal{K}\left(k_{4}, k_{5}, k_{6}, p\right)
\end{gathered}
$$

Clearly, these are hard, albeit doable, integrals. ${ }^{12}$ However, we will not dwell on these integrals for two reasons: the first reason is the simplicity of the computation of conformally coupled scalars compared to that of minimally coupled scalars. This is a fortunate observation because conformally coupled scalars can potentially be used as seed diagrams from which minimally coupled scalar can be computed as well [91]. ${ }^{13}$ And that is what we are turning to in the next section.

\section{CONFORMALLY COUPLED SCALARS}

\section{A. An algorithmic approach for conformally invariant scalars}

In Sec. II A we used a Weyl transformation to deduce which interaction terms preserve conformal invariance by going to the flat space and checking the form of interaction coefficient. However, we can use that Weyl transformation for computation purposes as well. Indeed, for conformally coupled scalars, ${ }^{14}$ the Lagrangian in Eq. (2.6) simplifies to

\footnotetext{
${ }^{12}$ One of the key points of [59] where the authors computed similar integrals for gluon exchange is that one can use residue theorem to significantly simplify such formidable integrals. Unfortunately, the integrands in Eq. (2.36) do not fall off at infinity hence residue theorem is no longer a simple option.

${ }^{13}$ In addition to those motivations, we also would like to note the claim of [92] that massive scalars in curved background may lie on the lightcone in the local Minkowski frame unless they are conformally coupled, leading to causal pathologies, indicating that any massive scalar needs to be conformally coupled.

${ }^{14}$ In the rest of the paper we mean $\xi=\xi_{c}$ and $m=0$ when we say conformally coupled scalar.
} 

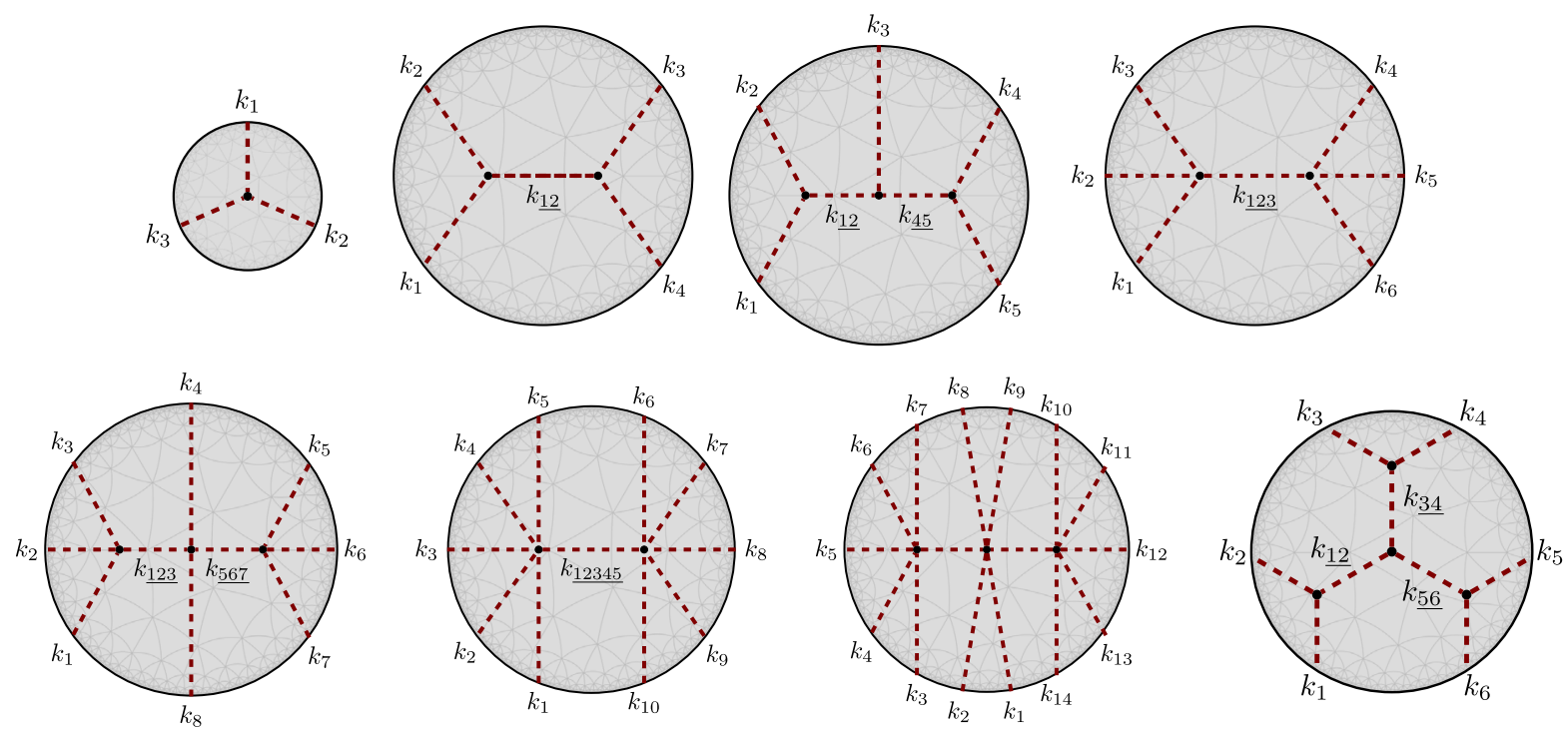

FIG. 2. Various tree level Witten diagrams that will be of interest below. From left to right, we label them as $W_{3,0}, W_{4,1}, W_{5,2}, W_{6,1}$, $W_{8,2}, W_{10,1}, W_{14,2}$, and $W_{6,3}$.

$$
S=-\int d^{d} x d z\left[\frac{1}{2}\left(\partial_{i} \phi\right)^{2}+\frac{1}{2}\left(\partial_{z} \phi\right)^{2}+\frac{\lambda_{n}(z)}{n !} \phi^{n}\right]
$$

under the transformation in Eq. (2.13). Here, we defined

$$
\lambda_{n}(z) \equiv \lambda_{n} z^{\frac{d-1}{2}\left(n-n_{c}\right)}
$$

where $i=1, \ldots, d$ run for the boundary coordinates with the boundary metric $\eta_{i j}$. From Eqs. (2.13b), (2.22b), (2.26), we can immediately write down the propagators: ${ }^{15}$

$$
\begin{gathered}
\phi_{k}(z)=\sqrt{\frac{2 k z}{\pi}} K_{1 / 2}(k z) \\
G_{\phi}\left(z, z^{\prime}, k_{i}\right)=\int_{0}^{\infty} \frac{-i p d p}{k_{i} k^{i}+p^{2}-i \epsilon} \\
\times\left(z^{1 / 2} J_{1 / 2}(p z)\right)\left(z^{1 / 2} J_{1 / 2}\left(p z^{\prime}\right)\right)
\end{gathered}
$$

from which we can deduce the bulk point integrations:

$$
\mathcal{B}_{n, x}(k, p) \equiv \int_{0}^{\infty} d z z^{\frac{d-1}{2}\left(n-n_{c}\right)} \prod_{i=1}^{x} \prod_{j=1}^{n-x} \phi_{k_{i}}(z)\left(z^{\frac{1}{2}} J_{\frac{1}{2}}\left(p_{j} z\right)\right) .
$$

Carrying out such integrals once and for all and then using those results in various different Witten diagrams is part of the strategies that were employed in $[59,60]$ as we

\footnotetext{
${ }^{15}$ As we explained in footnote 9 , we treat the overall $k$ dependence as normalization which we chose for the conformally coupled scalars in a consistent fashion with the similar work in $[35,36,62]$.
}

demonstrated in the case of minimally coupled scalars in Sec. II C. However, one can do better than calculating these integrals generically and using them case by case: we can directly find an algebraic algorithm and bypass all integrations, both the bulk-point $z$-integrations and propagator $p$-integrations.

Such an algorithm is discussed in [62] where the authors refer to the additive property of vertices, enabling them to work at the level of truncated diagrams and compute amplitudes directly via algebraic means. This is possible, as they argue, because the gluon propagators in $\mathrm{AdS}_{4}$ are simply exponentials and there is a nice cancellation between the volume factor $z^{-d-1}$ and the vertex factor $z^{4}$ in $\mathrm{AdS}_{4}$. We see that the propagators of conformally coupled scalars in flat space precisely match gluons in $\mathrm{AdS}_{4}$ and bulk point expressions have exactly same $z$-powers if $n=n_{c}$ as can be seen from Eq. (3.4). So we arrive at the conclusion that one can reduce the integrations to algebraic calculations for scalars with conformal symmetry in any dimension, analogous to gluons in $\mathrm{AdS}_{4}{ }^{16,17}$

Let us quickly review the algorithm to compute the Witten diagram amplitude of the form

\footnotetext{
${ }^{16}$ One can in fact still use the algorithm for conformally coupled scalars with $n \neq n_{c}$ with appropriate modification. We will discuss this in next section.

${ }^{17}$ This result is hardly surprising as the algorithm used in [62] is in fact derived by Arkani-Hamed et al. in [61] for conformally coupled scalars in dS. However the authors actually use the modified version of the algorithm that we will see in Sec. III B, hence they are not really trading all relevant integrations by an algebraic calculation. On the contrary, we will get rid of all integrals in this section, analogous to the case of gluons in $\mathrm{AdS}_{4}$.
} 


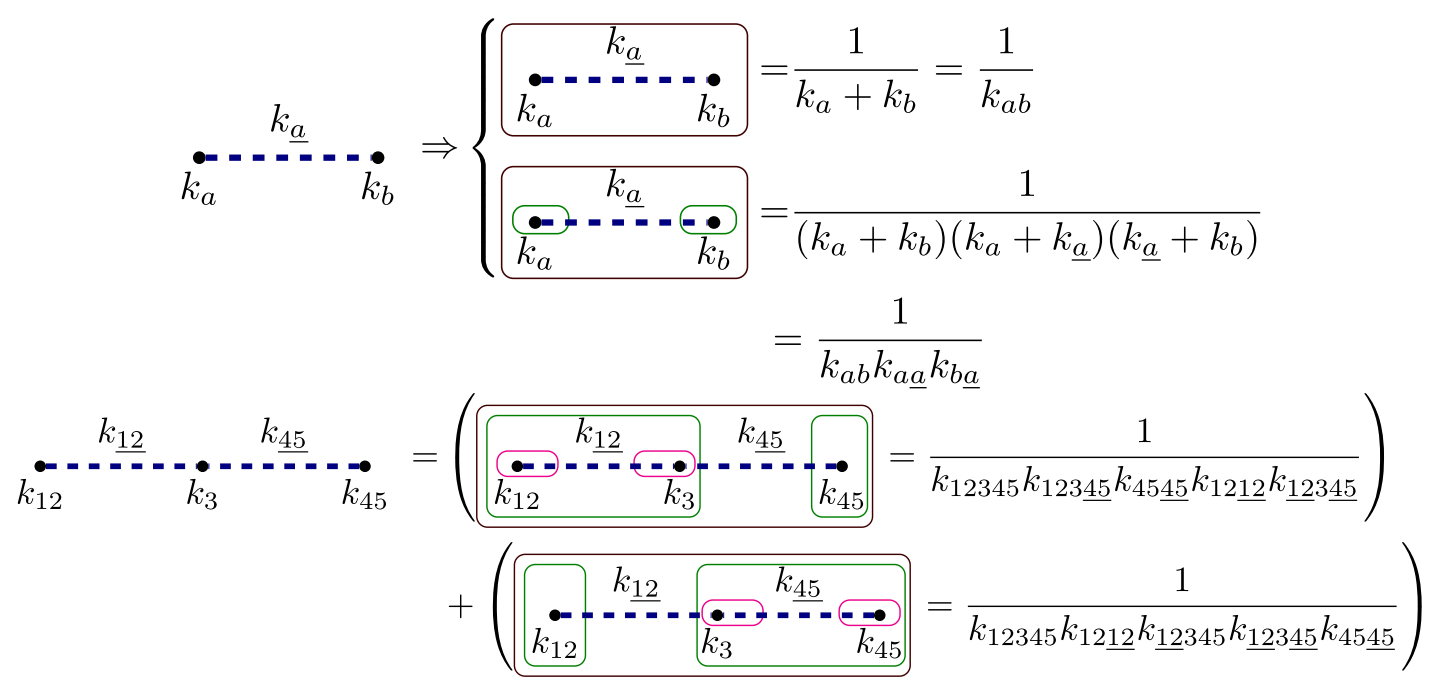

FIG. 3. Diagrammatic illustration of the algorithm.

$$
\begin{aligned}
W_{m, n} \equiv & \int_{0}^{\infty} d p_{1} \ldots d p_{n} \frac{-i p_{1}}{p_{1}^{2}+q_{1}^{2}-i \epsilon} \ldots \\
& \times \frac{-i p_{n}}{p_{n}^{2}+q_{n}^{2}-i \epsilon} \mathcal{B}_{n_{c}, x_{1}}\left(k_{i}, p_{i}\right) \ldots \\
& \times \mathcal{B}_{n_{c}, x_{n+1}}\left(k_{i}, p_{i}\right) \prod_{t=1}^{n+1}\left(-i \rho_{t}\right)
\end{aligned}
$$

which is the expression for the diagram of $m$ external legs $n$ bulk propagators (dependence on external legs is implicit in $\mathcal{B})$.

We note that the aforementioned additive property of the vertices, which follows from $\phi_{k_{1}}(z) \phi_{k_{2}}(z)=\phi_{k_{1}+k_{2}}(z)$, means that we can change the number of external legs as we wish as long as the sum of norms of the momenta flowing to vertices stay the same, up to the change in the coupling coefficients. Hence, we will work with the truncated diagram of the amplitude

$$
\mathcal{A}_{n} \equiv \frac{i^{2 n+1} W_{m, n}}{\prod_{t=1}^{n} \rho_{t}}
$$

which only depends on the topology of the truncated diagram, independent of the details of external legs but only the sum of norms of the incoming momenta. ${ }^{18}$ For example, for ${ }^{19} \mathcal{A}_{1}\left(k_{a}, k_{b}, k_{\underline{a}}\right)$, which is given in the first diagram of Fig. 3, the amplitudes of the four and six point

\footnotetext{
${ }^{18} \mathrm{We}$ introduced an additional $i^{n}$ factor for convenience; this way, our truncated amplitudes are exactly same with those of [62] in which $i$ factors are included in the projectors $\Pi$ instead of the scalar part of the propagator.

${ }^{19}$ In Figs. 3 and 5, we use the diagrammatic notation for truncated amplitudes in the same sense they are used in [62]: they correspond to Witten diagrams with bulk to boundary propagators stripped off.
}

diagrams shown in Fig. 2 for conformally coupled scalars read as

$$
\begin{aligned}
& W_{4,1}\left(k_{i}\right)=i \lambda_{3}^{2} \mathcal{A}_{1}\left(k_{12}, k_{34}, k_{\underline{12}}\right) \\
& W_{6,1}\left(k_{i}\right)=i \lambda_{4}^{2} \mathcal{A}_{1}\left(k_{123}, k_{456}, \underline{k_{123}}\right) .
\end{aligned}
$$

The algorithm for the computation of $\mathcal{A}$ is as follows. The diagram is decomposed into subdiagrams by cutting all internal lines. One then considers all possible orders in which the lines are cut, and assigns partial amplitudes to individual cases. The sum of these partial amplitudes give the full amplitude of the initial diagram.

The partial amplitude for a diagram with its lines cut in a particular order is the product of the amplitudes for all subgraphs, which are in turn equal to the inverse of the sum of all vertex norms within that subgraph and line norms going out of that subgraph.

In Fig. 3 we illustrate the algorithm for $\mathcal{A}_{1}$ and $\mathcal{A}_{2}$. For $\mathcal{A}_{1}$, we observe that there is only one partial amplitude which yields remarkably simple results

$$
\begin{aligned}
W_{4,1}\left(k_{i}\right) & =\frac{i \lambda_{3}^{2}}{k_{1234} k_{12 \underline{12}} k_{3412}}, \\
W_{6,1}\left(k_{i}\right) & =\frac{i \lambda_{4}^{2}}{k_{123456} k_{123 \underline{123}} k_{456123}}
\end{aligned}
$$

For $\mathcal{A}_{2}$, we have two partial amplitudes where the sum simplifies quite nicely, yielding

$$
\begin{aligned}
W_{5,2}\left(k_{i}\right) & =-i \lambda_{3}^{3} \mathcal{A}_{2}\left(k_{12}, k_{3}, k_{45}, k_{12}, k_{45}\right) \\
& =-i \lambda_{3}^{3} \frac{k_{12 \underline{12} 3345 \underline{45}}}{k_{12345} k_{12 \underline{12}} k_{345} \underline{12} k_{12} \underline{45} k_{4545} k_{12345} \underline{4}}
\end{aligned}
$$


We stated above that this algorithm is valid if $n=n_{c}$. By imposing $n \in \mathbb{Z}$ in Eq. (2.11), we see that there are only three cases with conformally invariant interactions: $\mathrm{AdS}_{3,4,6}$ with $\phi^{6,4,3}$ interaction. As the algorithm we provided is independent of the spacetime dimension, we can use it for all truncated diagrams; below we list some results for various Witten diagrams: one should understand the relevant dimension for which the amplitude is valid from the form of the interaction, i.e., results with 4-point interactions are valid for $\mathrm{AdS}_{4}$ only. ${ }^{20}$

$$
W_{10,1}\left(k_{i}\right)=i \lambda_{6}^{2} \mathcal{A}_{1}\left(k_{12345}, k_{6789(10)}, \underline{k_{12345}}\right)
$$

$$
\begin{gathered}
W_{8,2}\left(k_{i}\right)=-i \lambda_{4}^{3} \mathcal{A}_{2}\left(k_{123}, k_{48}, k_{567}, k_{\underline{123}}, k_{\underline{567}}\right) \\
W_{14,2}\left(k_{i}\right)=-i \lambda_{6}^{3} \mathcal{A}_{2}\left(k_{34567}, k_{1289}, k_{(10)(11)(12)(13)(14)}, k_{\underline{34567}},\right. \\
k_{\underline{(10)(11)(12)(13)(14)})}
\end{gathered}
$$

where

$$
\mathcal{A}_{1}\left(q_{1}, q_{2}, q_{3}\right)=\frac{1}{\left(q_{1}+q_{2}\right)\left(q_{1}+q_{3}\right)\left(q_{2}+q_{3}\right)}
$$

$$
\mathcal{A}_{2}\left(q_{1}, q_{2}, q_{3}, q_{4}, q_{5}\right)=\frac{\left(q_{1}+2 q_{2}+q_{3}+q_{4}+q_{5}\right)}{\left(q_{1}+q_{2}+q_{3}\right)\left(q_{1}+q_{4}\right)\left(q_{2}+q_{3}+q_{4}\right)\left(q_{2}+q_{4}+q_{5}\right)\left(q_{3}+q_{5}\right)\left(q_{1}+q_{2}+q_{5}\right)}
$$

We remind the reader of our notation given in Eq. (2.29). For example, $k_{(10)(11)(12)(13)(14)}$ above stands for $k_{10}+k_{11}+k_{12}+k_{13}+k_{14}$.

We would like to note that the method is not restricted to comblike diagrams, and can be used for other topologies as well. For example, for star diagram in Fig. 2, the algorithm yields

$W_{6,3}=(\mathcal{I}+34 \leftrightarrow 56)+\left(\begin{array}{l}12 \rightarrow 34 \\ 34 \rightarrow 56 \\ 56 \rightarrow 12\end{array}\right)+\left(\begin{array}{l}12 \rightarrow 56 \\ 56 \rightarrow 34 \\ 34 \rightarrow 12\end{array}\right)$

where

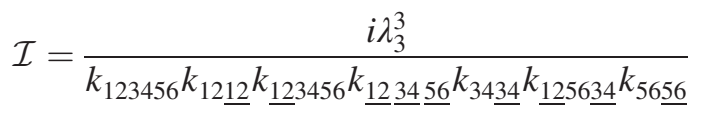

whose step by step computation can be found in [62].

\section{B. Generalized algorithm for all conformally coupled scalars}

There are not so many theories of interacting scalars with full conformal symmetry; in fact, the Eq. (2.11) tightly constraints the possibilities into three cases: $\mathrm{AdS}_{3}$ with $\phi^{6}$, $\mathrm{AdS}_{4}$ with $\phi^{4}$, and $\mathrm{AdS}_{6}$ with $\phi^{3}$ as we stated in previous section. However, we can extend our algorithm to all conformally coupled scalars which are not necessarily invariant under conformal transformations.

\footnotetext{
${ }^{20}$ We provide the results without dwelling on the relevance of the specific models. In particular, one can see $\phi^{3}$ potential in $\mathrm{AdS}_{6}$ as a mere toy model due to the $\mathbb{Z}_{2}$ odd potential yielding a Hamiltonian unbounded from below.
}

The restriction to conformally invariant scalars followed from the requirement to get rid of the additional $z$-factors in the bulk point integration in Eq. (3.4): we could use the algorithm if we were to expand additional $z$-factors in terms of $\phi_{k}(z)$. From Eq. (3.3a), we observe that this is indeed possible if we expand the interaction coefficients via Laplace transform, i.e. ${ }^{21}$

$$
\begin{aligned}
\tilde{\lambda}_{n}(\omega) & \equiv \int_{\gamma-i \infty}^{\gamma+i \infty} e^{\omega z} \lambda_{n}(z) \frac{d z}{2 \pi i}, \\
\lambda_{n}(z) & =\int_{0}^{\infty} e^{-\omega z} \tilde{\lambda}_{n}(\omega) d \omega=\int_{0}^{\infty} \phi_{\omega}(z) \tilde{\lambda}_{n}(\omega) d \omega .
\end{aligned}
$$

Thus, we can rewrite Eq. (2.27) as

$$
W_{m, n}=\int_{0}^{\infty} d \omega_{1} \ldots d \omega_{n+1} \prod_{t=1}^{n+1} \tilde{\rho}_{t}\left(\omega_{t}\right) \tilde{W}_{m, n}
$$

where $\tilde{\rho}_{m}$ is the appropriate $\tilde{\lambda}_{n}\left(\omega_{m}\right)$ at $m$ th vertex. Here, we defined

$$
\begin{aligned}
\tilde{W}_{m, n} \equiv & \int_{0}^{\infty} d z_{1} \ldots d z_{n+1} G_{\phi}\left(z_{j_{1}}, z_{j_{2}}, q_{1}\right) \ldots G_{\phi}\left(z_{j_{n}}, z_{j_{n}}, q_{n}\right) \\
& \times \phi_{k_{1}}\left(z_{i_{1}}\right) \ldots \phi_{k_{m}}\left(z_{i_{m}}\right) \phi_{\omega_{1}}\left(z_{1}\right) \ldots \phi_{\omega_{n+1}}\left(z_{n+1}\right)
\end{aligned}
$$

which exactly has the required form hence can be computed by mere algebraic means as we reviewed in the previous section.

Clearly, this modified algorithm is not as efficient as the original one because we still have to compute integrals to get the tree-level AdS amplitudes. However, for a Witten

\footnotetext{
${ }^{21}$ Here $\gamma$ is an arbitrary positive constant chosen so that the contour of integration lies to the right of all singularities in $\lambda_{n}(z)$.
} 
diagram of $n$ vertices, we are replacing $2 n-1$ integrations $^{22}$ with $n$ integrations whose integrand is computed algebraically; so this approach becomes rewarding especially as we consider higher order amplitudes. ${ }^{23}$

Apart from introducing a uniform treatment for all conformally coupled scalars, generalizing the algorithm as above can reveal algebraic and recursive relations between various Witten diagrams. In fact, this way of rewriting an amplitude is already done in [61] where they write the cosmological wave function $\tilde{\psi}$ for conformally coupled scalar as an integral over the modified wave function $\psi$ which follows from the Fourier expansion of the coupling coefficient $\lambda^{24}$ For the utility of such a representation in terms of algebraic and recursive means and relations with polytopes, we refer the reader to their paper.

As an example, we can consider $\phi^{3}$ interaction in $\mathrm{AdS}_{4}$. We can immediately read off $\tilde{W}$ from Eqs. (3.7) and (3.9) by including additional $\omega_{j}$ dependencies:

$$
\begin{aligned}
& \tilde{W}_{4,1}\left(k_{i}, \omega_{j}\right)=i \lambda_{3}^{2} \mathcal{A}_{1}\left(k_{12}+\omega_{1}, k_{34}+\omega_{2}, k_{12}\right) \\
& \tilde{W}_{5,2}\left(k_{i}, \omega_{j}\right)=-i \lambda_{3}^{3} \mathcal{A}_{2}\left(k_{12}+\omega_{1}, k_{3}+\omega_{2}\right. \text {, } \\
& \left.k_{45}+\omega_{3}, k_{12}, k_{45}\right)
\end{aligned}
$$

The amplitude for the relevant Witten diagrams become

$$
W_{4,1}=\int_{0}^{\infty} d \omega_{1} d \omega_{2} \tilde{W}_{4,1}, \quad W_{5,2}=\int_{0}^{\infty} d \omega_{1} d \omega_{2} d \omega_{3} \tilde{W}_{5,2} .
$$

Here we used the fact that the interaction coefficient is $\frac{-i \lambda_{3}}{z}$ whose numerator is taken into account in the calculation of $\tilde{W}$, hence

$$
\lambda_{3}(z)=z^{-1} \Rightarrow \tilde{\lambda}_{3}(\omega)=1 .
$$

Utilizing softwares for symbolic computations, such as Mathematica, we can calculate such integrals relatively easily. For example, $W_{4,1}$ reads as

\footnotetext{
${ }^{22} n-1 p$-integrations for the bulk to bulk propagators and $n z$-integrations as bulk-point integrations.

${ }^{23}$ One might object that this naive counting of integrals is misleading as we also need to compute $\tilde{\lambda}_{n}(\omega)$, the inverse Laplace transform of $\lambda_{n}(z)$. However, $\lambda_{n}(z)$ has a pure power law dependence for both polynomial and derivative interactions, hence its inverse Laplace transform is quite trivial, i.e. $\tilde{\lambda}_{n}(\omega)=\frac{\omega^{-1-k}}{\Gamma(-k)}$ for $\lambda_{n}(z)=z^{k}$.

${ }^{24}$ Our $W$ and $\tilde{W}$ are analogous to their $\tilde{\psi}$ and $\psi$ respectively. Likewise, their Eq. (2.9) is the analog of our (2.38).
}
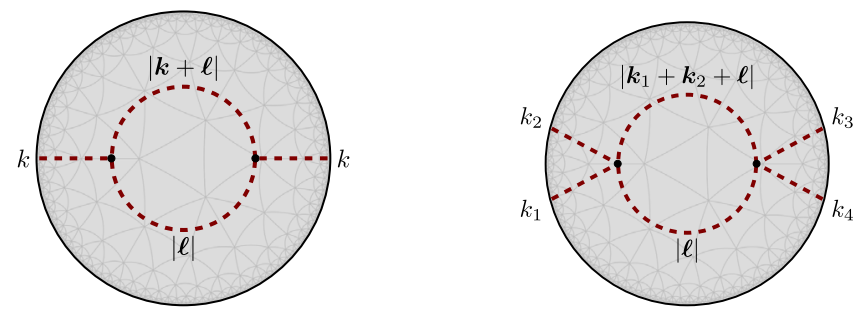

FIG. 4. One loop correction to the propagator and four point interaction in AdS.

$$
\begin{aligned}
W_{4,1}= & -\frac{i \lambda^{2}}{4 k_{\underline{12}}}\left(2 \operatorname{Li}_{2}\left(\frac{k_{1234}}{k_{12}-k_{\underline{12}}}\right)-2 \operatorname{Li}_{2}\left(\frac{k_{1234}}{k_{12 \underline{12}}}\right)\right. \\
& +\log ^{2}\left(\frac{1}{k_{\underline{12}}-k_{12}}\right)+\log ^{2}\left(k_{12 \underline{12}}\right) \\
& +2 \log \left(\frac{k_{\underline{12}}-k_{34}}{k_{12 \underline{12}}}\right) \log \left(\frac{k_{12 \underline{12}}}{k_{1234}}\right) \\
& -2 \log \left(k_{12 \underline{12}}\right) \log \left(k_{34 \underline{12}}\right) \\
& \left.+2 \log \left(k_{1234}\right) \log \left(\frac{k_{34 \underline{12}}}{k_{\underline{12}}-k_{12}}\right)+\pi^{2}\right) .
\end{aligned}
$$

One can compute other tree level expressions $W_{m, n}$ in a similar fashion.

\section{Extension to loops}

The algorithm we presented in Sec. III A, and its extension in Sec. III B readily applies to loops as well. In [61], such calculations are already done in the context of cosmological wave functions; here, we will illustrate the algorithm in the simplest case of one loop (see Fig. 4) with two vertices and use it to calculate 1 loop corrections to scalar amplitudes in AdS. ${ }^{25}$

The application of the algorithm in case of loops is same with that of tree level diagrams: we decompose the amplitude into sum of partial amplitudes where the expression for each partial amplitude follows from the vertex momenta inside the diagrams and the propagators going out of the diagrams. As we can see in Fig. 5, there are two partial amplitudes for one loop two vertex diagram. We multiply the corresponding expressions for each subdiagram in the denominator; we listed them from outward to inward: the first value, $\left(k_{a}+k_{b}\right)$, corresponds to the big circle, and the last ones correspond to small pink circles. In the end, the final expression takes a rather compact form in our notation: $\left(k_{a b} k_{\underline{a} a \underline{b}} k_{\underline{a b} \underline{b}}\right)^{-1}\left(k_{\underline{a} a b \underline{a}}^{-1}+k_{\underline{b} a b \underline{b}}^{-1}\right)$.

If we were dealing with a tree-level expression, we were done: we could simply translate from this truncated amplitude to a Witten diagram via Eq. (3.6). However, $k_{\underline{a}}$

\footnotetext{
${ }^{25} \mathrm{We}$ refer the reader to [93] for analysis of similar loop diagrams of conformally coupled scalars dual to vacuum correlator instead of transition amplitudes.
} 


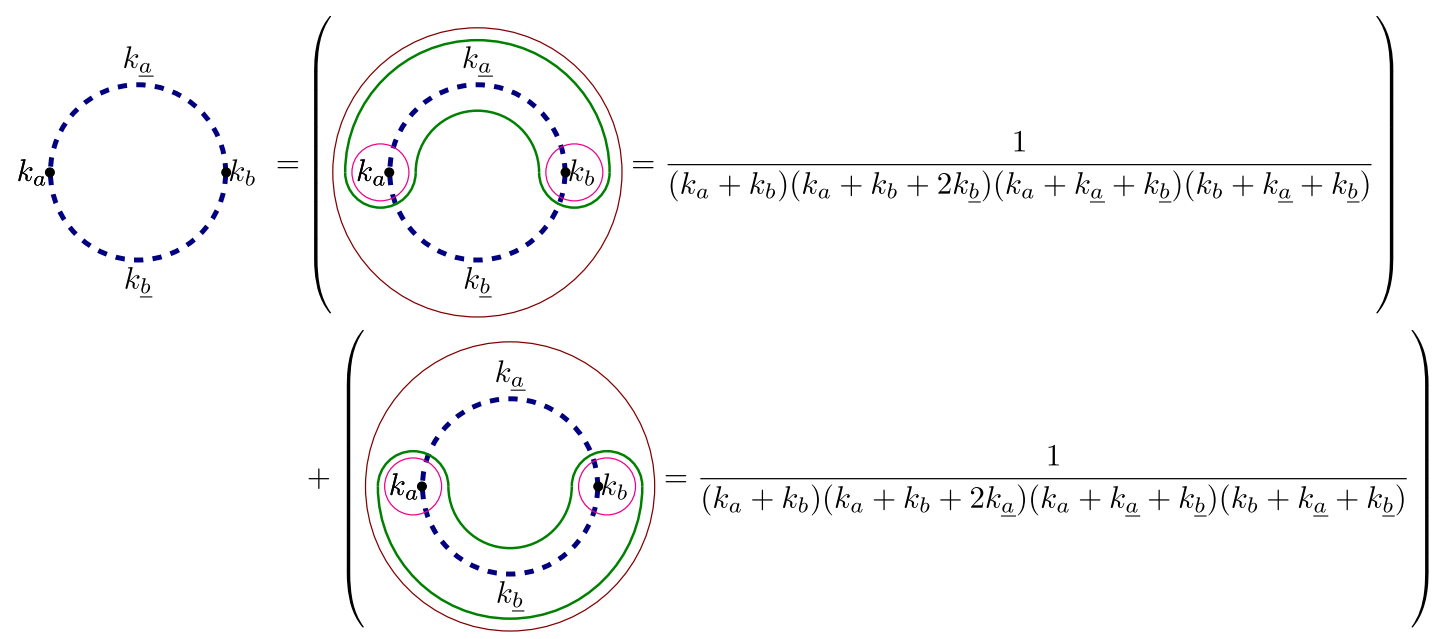

FIG. 5. The application of the algorithm to one loop two vertex diagram.

and $k_{b}$ depends on the loop amplitude and one needs to integrate it as well.

Let us illustrate this in case of loop correction to a two point function, the first diagram in Fig. 4, where we are assuming $\phi^{3}$ interaction in $\operatorname{AdS}_{6}{ }^{26}$ In this simple case the integrand becomes

$$
\begin{aligned}
\text { Integrand }= & \frac{1}{2 k(k+|\boldsymbol{l}|+|\boldsymbol{k}+\boldsymbol{l}|)^{2}} \\
& \times\left(\frac{1}{2 k+2|\boldsymbol{l}|}+\frac{1}{2 k+2|\boldsymbol{k}+\boldsymbol{l}|}\right)
\end{aligned}
$$

which is to be integrated over $l \in \mathbb{R}^{5}$. As the integration domain is invariant under successive applications of $\boldsymbol{l} \rightarrow-\boldsymbol{l}$ and $\boldsymbol{l} \rightarrow \boldsymbol{l}+\boldsymbol{k}$, which interchanges first and second term above, we can write the truncated amplitude as

$\mathcal{A}^{L}(k, k, \boldsymbol{k})=\int d^{5} \boldsymbol{l} \frac{1}{2 k(k+|\boldsymbol{l}|+|\boldsymbol{k}+\boldsymbol{l}|)^{2}(k+|\boldsymbol{l}|)}$

which reads in spherical coordinates after the Wick rotation as

$$
\begin{aligned}
& \mathcal{A}^{L}(k, k, \boldsymbol{k}) \\
& =-\frac{i S_{3}}{2 k} \int_{0}^{\pi} \sin \theta d \theta \\
& \quad \times \int_{0}^{\infty} l^{4} d l \frac{1}{\left(k+l+\sqrt{k^{2}+l^{2}+2 k l \cos \theta}\right)^{2}(k+l)}
\end{aligned}
$$

\footnotetext{
${ }^{26}$ As we saw in section, $d=5$ is the only case for which $\phi^{3}$ interaction can be calculated without additional $\omega$-integrations, which we would like to avoid to give the simplest example as the illustration.
}

where $S_{n}=\frac{2 \pi^{\frac{n+1}{2}}}{\Gamma\left(\frac{n+1}{2}\right)}$ is the volume of $n-$ sphere. We can carry out the $\theta$-integration immediately, yielding

$\mathcal{A}^{L}(k, k, \boldsymbol{k})=-\frac{i S_{3}}{2 k} \int_{0}^{\infty} d l \frac{l^{3}\left(-\frac{k+l}{|k-l|+k+l}+\log \left(\frac{2(k+l)}{|k-l|+k+l}\right)+\frac{1}{2}\right)}{k(k+l)}$.

Clearly, this is a divergent integral. By regularizing it with a hard cutoff $\Lambda,{ }^{27}$ we find

$$
\begin{aligned}
\mathcal{A}^{L}(k, k, \boldsymbol{k})= & -\frac{i \pi^{2}}{72}\left(\frac{18 \Lambda^{2}}{k}+96 k \log \left(\frac{\Lambda}{k}\right)\right. \\
& \left.+\left(39-6 \pi^{2}\right) k-72 \Lambda\right) .
\end{aligned}
$$

In the general case of $\phi^{n+2}$ interaction in $\operatorname{AdS}_{d+1}$, we have

$$
\begin{aligned}
k_{a} & =\left|\boldsymbol{k}_{1}\right|+\cdots+\left|\boldsymbol{k}_{n}\right|+\omega_{1} \\
k_{b} & =\left|\boldsymbol{k}_{n+1}\right|+\cdots+\left|\boldsymbol{k}_{2 n}\right|+\omega_{2} \\
k_{\underline{a}} & =|\boldsymbol{l}| \\
k_{\underline{b}} & =\left|\boldsymbol{l}+\boldsymbol{k}_{1}+\cdots+\boldsymbol{k}_{n}\right|
\end{aligned}
$$

which means $k_{\underline{a} a b \underline{a}} \leftrightarrow k_{\underline{b} a b \underline{b} \underline{b}}$ under the successive applications of $\boldsymbol{l} \rightarrow-\bar{l}$ and $\boldsymbol{l} \rightarrow \overline{\boldsymbol{l}}+\boldsymbol{k}_{1}+\cdots+\boldsymbol{k}_{n}$ under which the integration is invariant. Therefore, we have

\footnotetext{
${ }^{27}$ We thank Aaron Hillman for pointing out that one may need to choose $z$-dependent hard cutoff $\Lambda$ as energy scales vary with the bulk radius, and we believe that choosing $\Lambda / z$ should yield a more uniform energy cutoff. Nevertheless, our calculation should be fine for the purpose of illustrating the usage of algorithm with the loops.
} 


$$
\begin{aligned}
& \mathcal{A}^{L}\left(k_{a}, k_{b}, \boldsymbol{k}_{1}+\cdots+\boldsymbol{k}_{n}\right)=-\frac{2 i S_{d-2}}{k_{12 \ldots(2 n)}+\omega_{1}+\omega_{2}} \int_{0}^{\pi} \sin \theta d \theta \int_{0}^{\infty} l^{d-1} d l \frac{1}{k_{12 \ldots(2 n)}+\omega_{1}+\omega_{2}+2 l} \\
& \times \frac{1}{k_{12 \ldots n}+\omega_{1}+l+\sqrt{l^{2}+k_{\underline{12 \ldots n}}^{2}+2 l k_{12 \ldots n} \cos \theta}} \\
& \times \frac{1}{k_{(n+1)(n+2) \ldots(2 n)}+\omega_{2}+l+\sqrt{l^{2}+k_{\underline{12 \ldots n}}^{2}+2 l k_{\underline{12 \ldots n}} \cos \theta}}
\end{aligned}
$$

whose $\theta$ integration can be immediately carried out:

$$
\mathcal{A}^{L}\left(k_{a}, k_{b}, \boldsymbol{k}_{1}+\cdots+\boldsymbol{k}_{n}\right)=-\frac{4 i \pi^{\frac{d-1}{2}}}{\Gamma\left(\frac{d-1}{2}\right)} \times \int_{0}^{\infty} d l \frac{l^{d-2}\left(\left(k_{a}+l\right) \log \left(\frac{k_{a}+2 l+k_{12 \ldots n}}{k_{a}+\left|l-k_{12 \ldots n}\right|+l}\right)-\left(k_{b}+l\right) \log \left(\frac{k_{b}+2 l+k_{12 \ldots n}}{\left|l-k_{12 \ldots n}\right|+k_{b}+l}\right)\right)}{k_{12 \ldots n}\left(k_{a}-k_{b}\right)\left(k_{a}+k_{b}\right)\left(k_{a}+k_{b}+2 l\right)} .
$$

Despite the term $k_{a}-k_{b}$ in the denominator, the integrand above is actually continuous at $k_{a}=k_{b}$.

We were not able to compute the integration above for generic $d$, however, it is quite doable once we restrict to a specific $d$; for example,

$$
\begin{aligned}
& \left.\mathcal{A}^{L}\left(k_{a}, k_{b}, \boldsymbol{k}_{1}+\cdots+\boldsymbol{k}_{n}\right)\right|_{d=3}=-\frac{i \pi}{k_{a}+k_{b}}\left[\log (2)+\frac{k_{b} \log \left(\frac{k_{a}+k_{12 \ldots n}}{\Lambda}\right)-k_{a} \log \left(\frac{k_{b}+k_{12 \ldots n}}{\Lambda}\right)}{k_{a}-k_{b}}\right] \\
& -\frac{i \pi}{2 k_{\underline{12 \ldots n}}}\left[\operatorname{Li}_{2}\left(-\frac{k_{a}+k_{12 \ldots n}}{k_{b}-k_{\underline{12 \ldots n}}}\right)+\operatorname{Li}_{2}\left(-\frac{k_{b}+k_{12 \ldots n}}{k_{a}-k_{\underline{12 \ldots n}}}\right)+\log \left(k_{a}+k_{\underline{12 \ldots n}}\right) \log \left(k_{b}+k_{\underline{12 \ldots n}}\right)\right. \\
& -\log \left(k_{a}+k_{\underline{12 \ldots n}}\right) \log \left(k_{b}-k_{\underline{12 \ldots n}}\right)-\log \left(k_{a}-k_{\underline{12 \ldots n}}\right) \log \left(k_{b}+k_{\underline{12 \ldots n}}\right) \\
& \left.+\frac{1}{6}\left(3 \log ^{2}\left(k_{a}-k_{\underline{12 \ldots n}}\right)+3 \log ^{2}\left(k_{b}-k_{\underline{12 \ldots n}}\right)+\pi^{2}\right)\right] \text {. }
\end{aligned}
$$

Note that one still needs to integrate this result with respect to $\omega_{1}$ and $\omega_{2}$ by including appropriate $\tilde{\lambda}\left(\omega_{i}\right)$ factors. However, we know that $\phi^{4}$ interaction in $\mathrm{AdS}_{4}$ is actually conformally invariant so we do not need $\omega$ integrations if we focus on $\phi^{4}$ interaction. Indeed, we can directly write the full Witten expression for the second diagram in Fig. $4 \mathrm{as}^{28}$

$$
W=\lambda_{4}^{2} \mathcal{A}^{L}\left(k_{1}+k_{2}, k_{3}+k_{4}, \boldsymbol{k}_{1}+\boldsymbol{k}_{2}\right)
$$

\section{CONCLUSION}

In this paper, we have explored momentum space approach to computing amplitudes for scalars propagating in anti-de Sitter space. Adopting the algorithm provided in [61] for cosmological wave functions, we compute both tree and loop level examples of $\mathrm{AdS}$ transition amplitudes.

Our momentum space formalism provides a systematic and complementary study of scalars in AdS. We are interested in using this formalism to computing higher point scalar loop amplitudes, which we leave to a future study.

\footnotetext{
${ }^{28}$ This contradicts Eq. (3.6) because it is valid only for treelevel diagrams as we there make use of the fact that number of propagators is equal to number of vertices minus one.
}

Likewise, this formalism can be utilized for computation of spinning loops. Unlike scalars, computing gluon and graviton loops in general dimensions is complicated, but one can get nice results if one focuses on specific dimensions [94].

There are several promising avenues for further explorations. We believe that there should be a natural polytopic interpretation to the results that we have developed, parallel to the interpretation in [61]. It is also interesting whether the weight-shifting operators developed in [50] in the context of cosmology can be generalized into our formalism, allowing us to relate spinning momentum space amplitudes to scalar momentum space amplitudes. ${ }^{29}$ Lastly, momentum space approach has been used to construct crossing symmetric basis for CFT correlators [44,45]. Our approach could be useful in such explorations as well.

\section{ACKNOWLEDGMENTS}

S. A. is supported by DOE Award No. DE-SC0020318 and Simons Foundation Grant No. 488651. S. K. was supported by Divisional Research Funding Committee Discretionary Funds from Williams College.

\footnotetext{
${ }^{29}$ Such operators in positions space are constructed in [95].
} 
[1] J. M. Maldacena, The large N limit of superconformal field theories and supergravity, Int. J. Theor. Phys. 38, 1113 (1999); Adv. Theor. Math. Phys. 2, 231 (1998).

[2] E. Witten, Anti-de Sitter space and holography, Adv. Theor. Math. Phys. 2, 253 (1998).

[3] S. S. Gubser, I. R. Klebanov, and A. M. Polyakov, Gauge theory correlators from noncritical string theory, Phys. Lett. B 428, 105 (1998).

[4] D. Z. Freedman, S. D. Mathur, A. Matusis, and L. Rastelli, Comments on 4 point functions in the CFT/AdS correspondence, Phys. Lett. B 452, 61 (1999).

[5] H. Liu and A. A. Tseytlin, On four point functions in the CFT/AdS correspondence, Phys. Rev. D 59, 086002 (1999).

[6] D. Z. Freedman, S. D. Mathur, A. Matusis, and L. Rastelli, Correlation functions in the $\operatorname{CFT}(d) / \operatorname{AdS}(d+1)$ correspondence, Nucl. Phys. B546, 96 (1999).

[7] E. D'Hoker, D. Z. Freedman, and L. Rastelli, AdS/CFT four point functions: How to succeed at $z$ integrals without really trying, Nucl. Phys. B562, 395 (1999).

[8] E. D'Hoker, D. Z. Freedman, S. D. Mathur, A. Matusis, and L. Rastelli, Graviton exchange and complete four point functions in the AdS/CFT correspondence, Nucl. Phys. B562, 353 (1999).

[9] J. Penedones, Writing CFT correlation functions as AdS scattering amplitudes, J. High Energy Phys. 03 (2011) 025.

[10] M. F. Paulos, Towards Feynman rules for Mellin amplitudes in AdS/CFT, J. High Energy Phys. 10 (2011) 074.

[11] G. Mack, D-dimensional conformal field theories with anomalous dimensions as dual resonance models, Bulg. J. Phys. 36, 214 (2009).

[12] A. L. Fitzpatrick, J. Kaplan, J. Penedones, S. Raju, and B. C. van Rees, A natural language for AdS/CFT correlators, J. High Energy Phys. 11 (2011) 095.

[13] S. Kharel and G. Siopsis, Tree-level correlators of scalar and vector fields in AdS/CFT, J. High Energy Phys. 11 (2013) 159.

[14] A. L. Fitzpatrick and J. Kaplan, Analyticity and the holographic S-matrix, J. High Energy Phys. 10 (2012) 127.

[15] A. L. Fitzpatrick and J. Kaplan, Unitarity and the holographic S-matrix, J. High Energy Phys. 10 (2012) 032.

[16] M. S. Costa, V. Gonalves, and J. Penedones, Spinning AdS propagators, J. High Energy Phys. 09 (2014) 064.

[17] C. B. Jepsen and S. Parikh, Recursion relations in $p$-adic Mellin space, J. Phys. A 52, 285401 (2019).

[18] C. B. Jepsen and S. Parikh, p-adic Mellin amplitudes, J. High Energy Phys. 04 (2019) 101.

[19] S. S. Gubser, C. Jepsen, and B. Trundy, Spin in p-adic AdS/CFT, J. Phys. A 52, 144004 (2019).

[20] E. Hijano, P. Kraus, E. Perlmutter, and R. Snively, Witten diagrams revisited: The AdS geometry of conformal blocks, J. High Energy Phys. 01 (2016) 146.

[21] E. Y. Yuan, Simplicity in AdS perturbative dynamics, arXiv: 1801.07283.

[22] K. Ghosh, Polyakov-Mellin bootstrap for AdS loops, J. High Energy Phys. 02 (2020) 006.

[23] J. Liu, E. Perlmutter, V. Rosenhaus, and D. SimmonsDuffin, $d$-dimensional SYK, AdS loops, and $6 j$ symbols, J. High Energy Phys. 03 (2019) 052.

[24] S. Parikh, Holographic dual of the five-point conformal block, J. High Energy Phys. 05 (2019) 051.
[25] C. B. Jepsen and S. Parikh, Propagator identities, holographic conformal blocks, and higher-point AdS diagrams, J. High Energy Phys. 10 (2019) 268.

[26] J. Penedones, J. A. Silva, and A. Zhiboedov, Nonperturbative Mellin amplitudes: Existence, properties, applications, arXiv:1912.11100.

[27] F. Aprile, J. Drummond, P. Heslop, and H. Paul, One-loop amplitudes in $\mathrm{AdS}_{5} \times S^{5}$ supergravity from $\mathcal{N}=4 \mathrm{SYM}$ at strong coupling, J. High Energy Phys. 03 (2020) 190.

[28] D. Ponomarev, From bulk loops to boundary large-N expansion, J. High Energy Phys. 01 (2020) 154.

[29] D. Meltzer, AdS/CFT unitarity at higher loops: Highenergy string scattering, J. High Energy Phys. 05 (2020) 133.

[30] D. Meltzer, E. Perlmutter, and A. Sivaramakrishnan, Unitarity methods in AdS/CFT, J. High Energy Phys. 03 (2020) 061.

[31] S. Caron-Huot and A.-K. Trinh, All tree-level correlators in $\mathrm{AdS}_{5} \times \mathrm{S}_{5}$ supergravity: Hidden ten-dimensional conformal symmetry, J. High Energy Phys. 01 (2019) 196.

[32] S. Fichet, Opacity and effective field theory in anti-de Sitter backgrounds, Phys. Rev. D 100, 095002 (2019).

[33] S. Raju, BCFW for Witten Diagrams, Phys. Rev. Lett. 106, 091601 (2011).

[34] I. Mata, S. Raju, and S. Trivedi, CMB from CFT, J. High Energy Phys. 07 (2013) 015.

[35] S. Raju, Four point functions of the stress tensor and conserved currents in $\mathrm{AdS}_{4} / \mathrm{CFT}_{3}$, Phys. Rev. D 85, 126008 (2012).

[36] S. Raju, New recursion relations and a flat space limit for AdS/CFT correlators, Phys. Rev. D 85, 126009 (2012).

[37] S. Raju, Recursion relations for AdS/CFT correlators, Phys. Rev. D 83, 126002 (2011).

[38] N. Arkani-Hamed and J. Maldacena, Cosmological collider physics, arXiv:1503.08043.

[39] A. Bzowski, P. McFadden, and K. Skenderis, Implications of conformal invariance in momentum space, J. High Energy Phys. 03 (2014) 111.

[40] A. Bzowski, P. McFadden, and K. Skenderis, Scalar 3-point functions in CFT: Renormalisation, beta functions and anomalies, J. High Energy Phys. 03 (2016) 066.

[41] A. Bzowski, P. McFadden, and K. Skenderis, Renormalised CFT 3-point functions of scalars, currents and stress tensors, J. High Energy Phys. 11 (2018) 159.

[42] A. Bzowski, P. McFadden, and K. Skenderis, Evaluation of conformal integrals, J. High Energy Phys. 02 (2016) 068.

[43] H. Isono, T. Noumi, and T. Takeuchi, Momentum space conformal three-point functions of conserved currents and a general spinning operator, J. High Energy Phys. 05 (2019) 057.

[44] H. Isono, T. Noumi, and G. Shiu, Momentum space approach to crossing symmetric CFT correlators, J. High Energy Phys. 07 (2018) 136.

[45] H. Isono, T. Noumi, and G. Shiu, Momentum space approach to crossing symmetric CFT correlators. Part II. General spacetime dimension, J. High Energy Phys. 10 (2019) 183.

[46] C. Corianò and M. M. Maglio, Exact correlators from conformal ward identities in momentum space and the perturbative $T J J$ vertex, Nucl. Phys. B938, 440 (2019). 
[47] C. Corianò, M. M. Maglio, A. Tatullo, and D. Theofilopoulos, Exact correlators from conformal ward identities in momentum space and perturbative realizations, Proc. Sci., CORFU2018 (2019) 072 [arXiv:1904.13174].

[48] C. Corianò and M. M. Maglio, On some hypergeometric solutions of the conformal ward identities of scalar 4-point functions in momentum space, J. High Energy Phys. 09 (2019) 107.

[49] M. Gillioz, Momentum-space conformal blocks on the light cone, J. High Energy Phys. 10 (2018) 125.

[50] N. Arkani-Hamed, D. Baumann, H. Lee, and G. L. Pimentel, The cosmological bootstrap: Inflationary correlators from symmetries and singularities, J. High Energy Phys. 04 (2020) 105.

[51] C. Corianò and M. M. Maglio, The general 3-graviton vertex (TTT) of conformal field theories in momentum space in $d=4$, Nucl. Phys. B937, 56 (2018).

[52] C. Corianò, L. Delle Rose, E. Mottola, and M. Serino, Solving the conformal constraints for scalar operators in momentum space and the evaluation of Feynman's master integrals, J. High Energy Phys. 07 (2013) 011.

[53] A. Bzowski, P. McFadden, and K. Skenderis, Conformal $n$-Point Functions in Momentum Space, Phys. Rev. Lett. 124, 131602 (2020).

[54] N. Anand, Z. U. Khandker, and M. T. Walters, Momentum space CFT correlators for Hamiltonian truncation, arXiv:1911.02573.

[55] M. Gillioz, Conformal 3-point functions and the Lorentzian OPE in momentum space, arXiv:1909.00878.

[56] J. A. Farrow, A. E. Lipstein, and P. McFadden, Double copy structure of CFT correlators, J. High Energy Phys. 02 (2019) 130.

[57] A. Lipstein and P. McFadden, Double copy structure and the flat space limit of conformal correlators in even dimensions, arXiv:1912.10046 [Phys. Rev. D (to be published)].

[58] B. Nagaraj and D. Ponomarev, Spinor-Helicity formalism for massless fields in $\mathrm{AdS}_{4}$ II: Potentials, arXiv: 1912.07494.

[59] S. Albayrak and S. Kharel, Towards the higher point holographic momentum space amplitudes, J. High Energy Phys. 02 (2019) 040.

[60] S. Albayrak and S. Kharel, Towards the higher point holographic momentum space amplitudes II: Gravitons, J. High Energy Phys. 12 (2019) 135.

[61] N. Arkani-Hamed, P. Benincasa, and A. Postnikov, Cosmological polytopes and the wavefunction of the Universe, arXiv:1709.02813.

[62] S. Albayrak, C. Chowdhury, and S. Kharel, New relation for Witten diagrams, J. High Energy Phys. 10 (2019) 274.

[63] J. M. Maldacena, Non-Gaussian features of primordial fluctuations in single field inflationary models, J. High Energy Phys. 05 (2003) 013.

[64] A. Ghosh, N. Kundu, S. Raju, and S. P. Trivedi, Conformal invariance and the four point scalar correlator in slow-roll inflation, J. High Energy Phys. 07 (2014) 011.

[65] P. McFadden and K. Skenderis, Holography for cosmology, Phys. Rev. D 81, 021301 (2010).

[66] X. Chen and Y. Wang, Quasi-single field inflation and non-Gaussianities, J. Cosmol. Astropart. Phys. 04 (2010) 027 .
[67] J. M. Maldacena and G. L. Pimentel, On graviton nonGaussianities during inflation, J. High Energy Phys. 09 (2011) 045.

[68] D. Baumann and D. Green, Signatures of supersymmetry from the early Universe, Phys. Rev. D 85, 103520 (2012).

[69] V. Assassi, D. Baumann, and D. Green, On soft limits of inflationary correlation functions, J. Cosmol. Astropart. Phys. 11 (2012) 047.

[70] X. Chen and Y. Wang, Quasi-single field inflation with large mass, J. Cosmol. Astropart. Phys. 09 (2012) 021.

[71] V. Assassi, D. Baumann, D. Green, and L. McAllister, Planck-suppressed operators, J. Cosmol. Astropart. Phys. 01 (2014) 033.

[72] H. Lee, D. Baumann, and G. L. Pimentel, Non-Gaussianity as a particle detector, J. High Energy Phys. 12 (2016) 040.

[73] H. An, M. McAneny, A. K. Ridgway, and M. B. Wise, Quasi single field inflation in the non-perturbative regime, J. High Energy Phys. 06 (2018) 105.

[74] A. Kehagias and A. Riotto, On the inflationary perturbations of massive higher-spin fields, J. Cosmol. Astropart. Phys. 07 (2017) 046.

[75] S. Kumar and R. Sundrum, Heavy-lifting of gauge theories by cosmic inflation, J. High Energy Phys. 05 (2018) 011.

[76] D. Baumann, G. Goon, H. Lee, and G. L. Pimentel, Partially massless fields during inflation, J. High Energy Phys. 04 (2018) 140.

[77] G. Franciolini, A. Kehagias, and A. Riotto, Imprints of spinning particles on primordial cosmological perturbations, J. Cosmol. Astropart. Phys. 02 (2018) 023.

[78] G. Goon, K. Hinterbichler, A. Joyce, and M. Trodden, Shapes of gravity: Tensor non-Gaussianity and massive spin-2 fields, J. High Energy Phys. 10 (2019) 182.

[79] D. Anninos, V. De Luca, G. Franciolini, A. Kehagias, and A. Riotto, Cosmological shapes of higher-spin gravity, J. Cosmol. Astropart. Phys. 04 (2019) 045.

[80] S. Pi and M. Sasaki, Curvature perturbation spectrum in two-field inflation with a turning trajectory, J. Cosmol. Astropart. Phys. 10 (2012) 051.

[81] J.-O. Gong, S. Pi, and M. Sasaki, Equilateral nonGaussianity from heavy fields, J. Cosmol. Astropart. Phys. 11 (2013) 043.

[82] C. Sleight, A Mellin space approach to cosmological correlators, J. High Energy Phys. 01 (2020) 090.

[83] C. Sleight and M. Taronna, Bootstrapping inflationary correlators in Mellin space, J. High Energy Phys. 02 (2020) 098.

[84] A. Hillman, Symbol recursion for the dS wave function, arXiv:1912.09450.

[85] S. Sonego and V. Faraoni, Coupling to the curvature for a scalar field from the equivalence principle, Classical Quantum Gravity 10, 1185 (1993).

[86] N. D. Birrell and P. C. W. Davies, Quantum Fields in Curved Space, Cambridge Monographs on Mathematical Physics (Cambridge University Press, Cambridge, England, 1984).

[87] G. W. Gibbons and S. W. Hawking, Action integrals and partition functions in quantum gravity, Phys. Rev. D 15, 2752 (1977).

[88] A. Guarnizo, L. Castaneda, and J. M. Tejeiro, Boundary term in metric $f(R)$ gravity: Field equations in the metric formalism, Gen. Relativ. Gravit. 42, 2713 (2010). 
[89] S. Nojiri and S. D. Odintsov, Brane world cosmology in higher derivative gravity or warped compactification in the next-to-leading order of AdS/CFT correspondence, J. High Energy Phys. 07 (2000) 049.

[90] D. Carmi, Loops in AdS: From the spectral representation to position space, arXiv:1910.14340.

[91] D. Baumann, C. Duaso Pueyo, A. Joyce, H. Lee, and G. L. Pimentel, The cosmological bootstrap: Weight-shifting operators and scalar seeds, arXiv:1910.14051.
[92] V. Faraoni, Conformally coupled inflation, Galaxies 1, 96 (2013).

[93] S. Giombi, C. Sleight, and M. Taronna, Spinning AdS loop diagrams: Two point functions, J. High Energy Phys. 06 (2018) 030.

[94] S. Albayrak, C. Chowdhury, and S. Kharel (to be published).

[95] M. S. Costa and T. Hansen, AdS weight shifting operators, J. High Energy Phys. 09 (2018) 040. 